\title{
Recent Approaches to Enhance the Efficiency of Ultra-Wide Band MAC Protocols
}

\author{
Mohamed Ali Ahmed \\ College of Community, University of \\ Hail, Hail, Saudi Arabia. \\ Faculty of Science, Suez Canal \\ University, Egypt.
}

\author{
H. M. Bahig \\ College of Computer Science and \\ Engineering, University of Hail, \\ Hail, Saudi Arabia. \\ Ain Shams University, Cairo, Egypt
}

\author{
Hassan Al-Mahdi \\ Al Jouf University, \\ Saudi Arabia
}

\begin{abstract}
Ultra-wide band (UWB) is a promising radio technology to transmit huge data in short distances between different digital devices or between individual components of a personal computer. Due to the magnificent features of UWB technology, it finds vast research and application interests, such as Wireless Personal Area Networks (WPANs), Wireless Sensor Networks (WSNs), Wireless Body Area Networks (WBANs) as a special case of WSNs, and Wireless Area Networks (WLANs) as well. In this article, we study the assumptions and performance metrics related to recent schemes of Medium Access Control (MAC) Protocols employed in UWB applications that aim to improve its performance. Also, we compare the different approaches used in the recent works based on 10 parameters: application domain, cast type, protocol centralization, number of hops, mobility, number of used channels, uniformity, priority, and analytical approach. Finally, we introduce different approaches to improve UWB applications.
\end{abstract}

Keywords-Ultra-wide band (UWB); Wireless Personal Area Network (WPAN); Wireless Sensor Network (WSN); Wireless Body Area Network (WBAN); Medium Access Control (MAC); performance metrics

\section{INTRODUCTION}

Huge sizes of data are generated due to the great advances in both hardware and applications. For example, new digital cameras create high-quality images and videos. These images and videos data are required to be transmitted from a camera or smart TV to different devices in a short range. A simple solution is to use cables via Universal Serial Bus (USB) or High-Definition Multimedia Interface (HDMI) ports. However, in many cases, using cables is not the good idea, especially in connecting smart devices or computers to each other. Using wireless connections provide more pliability to install rapid communications. Therefore, it is a significant objective to introduce a suitable secure transmission technology with high transmission rate in short distance communications to send data between different nodes (either components or devices). Ultra-Wide Band (UWB) technology is a suitable approach to attain this objective.

UWB is mostly different from other communication technologies. It depends mainly on utilizing radio waves with short pulses or impulses radio, alternatively. When the duration of the generated pulses is decreased, the bandwidth of the transmission spectrum is increased. Therefore, communicating nodes transfer data with high transmission rates.

Many applications employ wireless technologies to implement UWB communication such as WPANs, WLANs, WSNs, WBANs, Wireless USB, Next-generation of Bluetooth, peer-to-peer connections, and Worldwide Interoperability for Microwave Access (WiMAX). In this article, we will focus on WPANs, WSNs, and WBANs. However, there are little applications employ wire-based approaches to implement UWB communication.

Due to the wide spectrum of UWB signals, many users (devices or nodes) require to share medium between different users. A special care is needed to control access of the shared medium efficiently. Therefore, Medium Access Control (MAC) sublayer is widely addressed area in performance enhancement and analyzing of UWB technology. MAC sublayer is a part of the data link layer which contains protocols that are responsible for controlling medium sharing among many users. MAC is an important research area among those addressed by many researchers.

Different fertile research areas are introduced for UWB such as generation of UWB signals, modulation, transmission, medium access, power saving, and secure communication. Also, introducing new applications that fit the characteristics of UWB is a promising goal for modern research works.

The main goal of this work is to study the recent approaches that are used to enhance the UWB technology performance via MAC sublayer's protocols for many applications including WPANs, WBANs and WSNs.

Studying those approaches precisely is an important objective to determine which of the addressed enhancements can be applied to a specific situation. Also, it is important to discover the shortage of a specific domain to be enhanced later according to specific criteria.

The remainder of the paper is organized as follows. Section 2 introduces the definition of UWB, its characteristics, advantages, disadvantages, applications, and finally, states a set of performance metrics used in evaluating the performance of a specific UWB scheme. Section 3 introduces the current approaches in performance improvement of UWB applications. Section 4 compares the different works according to many parameters. Next, some ideas and future 
work areas to measure or improve the performance of UWB applications are suggested. Finally, Section 5 concludes the work.

\section{OVERVIEW OF UWB}

This section briefly discusses the most significant topics related to UWB technology. These topics include UWB definition, characteristics, advantages, disadvantages, and applications. Finally, the most important performance metrics used to assess the efficiency of a specific UWB scheme are stated.

According to Federal Communications Commission (FCC) regulations, UWB is defined as a wide-band transmission technology having a signal of a fractional bandwidth larger than $20 \%$ of its central frequency, or simply UWB is a transmission technology having bandwidth more than 500 $\mathrm{MHz}$ [1].

UWB technology has many characteristics that give it an advantage and a higher priority in both commercial and research fields to increase and improve its applications.

The main characteristics that distinguish UWB technology are: (1) It has a great ability to be used in short-range wireless applications. (2) It covers small ranges from 7 up to about 30 meters. However, the increase in coverage range implies to decrease in speed. (3) It has a high data rate, where the data rate of UWB can reach to $1.3 \mathrm{Gbps}$ in some cases [2]. (4) It has different operating frequencies: below $1 \mathrm{GHz}, 3-5 \mathrm{GHz}$ and 6-10 GHz. However, the frequency range $(3.1-10.6 \mathrm{GHz})$ is commonly used [3] and [4]. (5) It works according to many standards. The common IEEE standards that UWB uses are IEEE 802.15.3, IEEE 802.15.4, IEEE 802.15.4a for UWB WPANs [5], IEEE 802.15.4a for UWB sensor networks [6] and IEEE 802.15.6 for WBANs [7], and [8].

UWB has many advantages such as (1) it uses low power. (2) It has good noise immunity. (3) It has signals of a great ability to penetrate different materials easily. (4) It has very high transmission rates. (5) It has a low probability of intercept and detection.

The main disadvantages of UWB are as follows: (1) It needs high technology components to work properly. (2) It has a high cost. (3) It has long signal acquisition times.

One of the important applications employing UWB is WPANs. WPAN is a wireless short range network used to link computer components to the main computer. Usually, WPAN covers a distance from 7 to 13 meters with 10 meters distance average. Also, WPANs use Bluetooth as a common specification that determines the communication parameters for wireless connections among portable computer's components, digital devices such as smart mobile phones, and/or notebooks. A typical case of a WPAN is shown in the Fig. 1.

Besides, WPAN can be used to connect different digital devices without using computers. UWB is an ascendant approach used instead of Bluetooth to transmit data in
WPANs at high speed. WLANs [9] can be seen as a generalization of WPANs. Also, WLANs utilize UWB technology to increase the transmission speed between individual computers and employ IEEE-802.11a standard [10], [11] and [12].

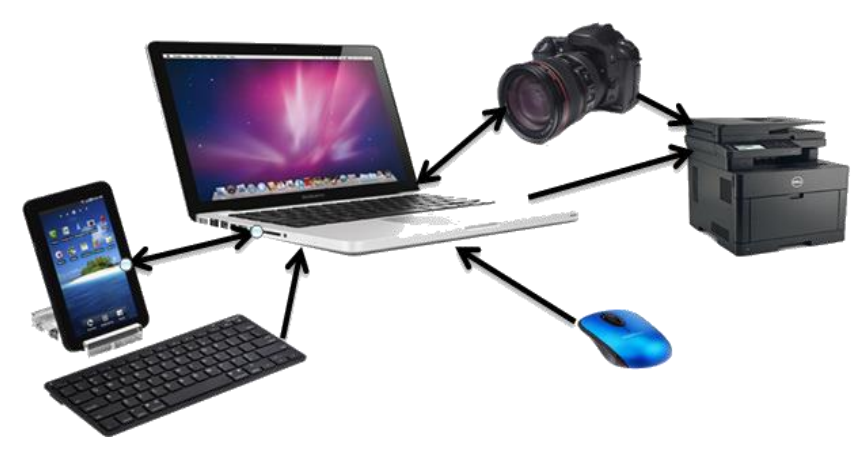

Fig. 1. A typical WPAN construction.

A WSN is a network that connects individual sensor devices, where each device is responsible for gathering specific data by sensing the surrounding medium. WSNs are used in many applications including emergency, management, smart home and city, better society, industry, weather forecasting, traffic monitoring, reveal fires inside home or forests and health. Actually, WBAN is a special purpose network of WSN for using sensors in the health domain. It is used to link sensors installed, inside and/or outside, in different organs of a human's body.

Many approaches were introduced to improve the efficiency of UWB technology such as improving antennas or chips [13], modulation techniques [14], MAC sublayer to adjust and control the media access [15]-[[20], security [21] and [22], localization [23], [24], and [25], and power control [25] and [26].

Different performance metrics are used to evaluate the efficiency of a specific scheme as follows. (1) Block probability: number of data packets lost due to buffer full. (2) Drop probability: number of data packets lost from buffer head resulting from the expiration of the number of retransmission trials. (3) Loss probability: number of all lost data packets resulting from either block probability or drop probability. (4) Delay: the total time needed to transmit a data packet from source node to a destination node. (5) Power consumption: the power used to perform networking activity. (6) Throughput: the number of successfully transmitted data packets. (7) Bit error rate: the rate of unsuccessfully transmitted data bits due to noise or any other reason. (8) Mean service time: the average time required to transmit the head of queue data packet from source to destination. (9) Medium utilization: the time percentage the bandwidth successfully used. (10) Reliability: delivery of data to the willful recipient(s) with a high level of assurance. (11) Jitter: the delay variation of packets receiving. (12) Detection probability: the probability of detecting a certain node by neighbor nodes. 


\section{AdVANCES IN MAC PROTOCOLS OF UWB APPLICATIONS}

This section investigates the recent suggested works to improve the efficiency of UWB MAC sublayer of many applications including WPANs and others.

Backoff algorithms have a great role in improving the performance of MAC protocols. Khan et al. [18] presented a novel algorithm named Prioritized Fibonacci Backoff (PFB) to improve the performance of the protocol and to replace both algorithms: Binary Exponential Backoff (BEB) and Alternative Binary Exponential Backoff (ABEB). Consequently, they constructed an analytical model for carrier sense multiple access with collision avoidance CSMA/CA mechanism of IEEE 802.15.6.

In the proposed model, the node state is modelled with Discrete Time Markov Chain (DTMC) with priority traffic and can be either homogeneous or heterogeneous. The numerical results show that the proposed $\mathrm{PFB}$ is more efficient than the ABEB.

Wei et al. [27] proposed an enhanced channel hopping scheme to allow multiple WBANs to communicate with each other using the same channel. The authors introduced a set of analysis approaches by taking the queuing and non-queuing behaviours into consideration to evaluate the coexistence capability, and the maximum number of WBANs that each channel supports. Also, they presented both service-dependent and service-independent analysis models to estimate the actual number of coexisting WBANs. When WBANs detect a coexistence conflict, the channel hopping mechanisms select a new channel according to distributed or centralized, orderly or randomly, event or time-driven analysis models. The results show that the enhanced channel hopping mechanism significantly improves the coexistence capability and allow larger arrival rates of WBANs.

Sudjai et al. [9] proposed an adaptive ultra-wideband WBAN to enhance the average bit error efficiency for high data traffic applications with an acceptable high throughput in a body to external links of that WBAN.

The proposed network utilizes a multiband orthogonal frequency division multiplexing (MB-OFDM) assuming space-time-frequency-coded (STFC) ultra-wideband technology to enhance the average bit error performance of external links of a WBAN. The proposed model utilizes two novel components in comprise: the frame transmission model, and the bit error rate (BER) estimation-based adaptive algorithm.

The proposed adaptive scheme depends on measuring the quality of BER of a signal at receivers. The measured signal will be compared to the signal thresholds. Based on the signal thresholds, three modulation sets are defined, each set has different modulation scheme. The decoding process of the first set depends on the 3/2-rate STFC and Quadrature Phase Shift Keying (QPSK) code modulation. The second set depends on a QPSK modulator with the full-rate Alamouti code as the STFC. The third set utilizes the Alamouti code. Results show that the suggested model reduces the consumption of power compared to the non-optimized adaptive WBAN and the nonadaptive WBAN system.

Kim et al. [8] suggested a prioritized resource allocation algorithm which is basically designed to capture the features of an active superframe interleaving scheme. The suggested algorithm has two major contributions. (1) Improving network performance using a single channel like FDM. (2) Improving the compatibility of different devices by utilized IEEE 802.15.6. In the suggested scheme, each WBAN specifies the required channel resource using the fluid twin token bucket TSPEC model. Many formulas to find the values of peak service rate, minimum service rate and maximum allowed delay were introduced. WBANs with high priority increases Quality of Service (QoS) for medical and health services.

Guizar et al. [15] assumed mobile nodes WBAN. They aimed to estimate its relative positions using known positions on-body anchors according to realistic channel models. To achieve this goal, the authors implemented three channel models based on the following: (1) the theoretical path loss of on-body nodes defined by the Channel Model 3 Impulse Radio UWB (3-10 GHz), (2) the channel model is simulated by a ray-tracing (PyLayers), and (3) an experimental channel obtained by measurement.

In the proposed work, authors aimed to alleviate the positioning loss via cross-layer distributed-cooperative algorithm depends on two link quality estimators: 1) a longterm Link Quality Estimators LQEs to identify the anchors introducing low ranging rate with the nodes, 2) a short-term LQEs to analyze reliable links with other nodes in order to replace the failing anchors. They evaluated the positioning success rate with different MAC scheduling based on Time Division Multiple Access (TDMA).

The authors considered a fully connected WBAN where all nodes can directly communicate in pairs to perform the motion capture. They defined two on-body types of sensors: 1) "On-body anchor sensors" that have knowledge of their absolute and relative positions at any time. 2) "On-body mobile sensors" which want to estimate their relative positions.

The authors concluded their work as the proposed algorithm leads to an increase of positioning success rate for all nodes with the worst localization performance for most of the scheduling strategies and different channel models.

El Azhari et al. [17] discussed the architecture system of WBAN and the details of IEEE 802.15.6 standard. Also, they studied the effect of WBANs on channel propagation. The 
authors introduced superframe structure used by the MAC sublayer to find the average end-to-end delay, channel fading, energy consumption, and packet reception. Also, the authors studied both single-hop networking according to centralized MAC protocol and multi-hop with distributed MAC protocol. Finally, the authors used simulation to evaluate the performance of the introduced model.

Kumar et al. [16] developed a mathematical model with a discrete-time Markov chain (DTMC) to significantly evaluate the performances of IEEE 802.15.6 CSMA/CA with non-ideal channel condition having high traffic rates. Authors developed a 4-tuple DTMC model based on user priority, backoff stages, backoff counter state, and state of retransmission counter at a time. They also included the failure probability due to collision by distinctively modelling the acknowledgement. Finally, they computed collision probability, end-to-end delay, average processing time, reliability, throughput, CCA (circuit card assembly) failure probability, and packet drop probability.

Ding et al. [29] studied the efficiency of UWB using two standard MAC protocols: the distributed coordination protocol with CSMA/CA and the centralized protocol with TDM. They applied both protocols to UWB WLAN and studied the acquisition times on overall performance in terms of the size of packet frames and rates of packet arrival. The synchronization time between both transmitter and receiver usually be quite because of high the pico-second precision pulses used in both UWB and impulse radio. Between many assumptions, authors assumed that channel BER is zero.

To study the performance of the proposed protocols, the authors compared CSMA/CA and TDMA protocols to narrowband and wideband channels. Additionally, they used utilization, delay, and throughput to evaluate the system performance. The results show that CSMA/CA and TDMA are not suitable for UWB networks, compared to narrowband and wideband networks. The main reason for this drawback is the longer channel acquisition times.

Joo et al. [26] proposed an efficient resource reservation scheme using cross-layer link adaptation for Ultra-Wide Band WPAN with Distributed Medium Access Control (D-MAC) to be used in Wi-Media. The authors proposed Relay Distributed Reservation Protocol (DRP) with relay node selection criteria depending on making a relay path.

For interoperability with the existing Wi-Media D-MAC standard, the proposed Relay DRP just utilized the reserved fields of the current Wi-Media D-MAC specification. To support the legacy Wi-Media D-MAC with the new functionality of cooperative relay transmission, the Relay DRP used three new code-points for resource reservation of cooperative data relay instead of the reserved fields of the current Wi-Media D-MAC standard.

Simulation results show that the proposed method has an enhancement for throughput and energy consumption. Also, the simulation results show that the Relay DRP scheme is compatible and applicable directly with a small overhead to the current Wi-Media D-MAC standard system.

Shi et al. [30] introduced an Average Time Slot Multiplexing Allocation (ATSMA) algorithm based on Space/Time Division Multiple Access (STDMA). The main contributions of authors' work include two parts. (1) Establishing a device interconnected algorithm of $60 \mathrm{GHz}$ WPAN system based on switched beam code to guarantee free switching between the devices under directional communication. (2) Enhancing multiple access under 802.15.3c standard. The authors plugged STDMA into the 802.15.3c standard. In the case of certain time slot allocation fairness the ATSMA algorithm enhances the capacity of the $60 \mathrm{GHz}-$ WPAN system significantly.

Park et al. [31] improved the average overall throughput and followed in mmWave directional networks by suggesting a new algorithm, called Multi-hop Opportunistic Concurrent Directional Transmission (M-OCDT) based on IEEE 802.15.3c standard. The proposed algorithm provides directional multicast communication by using relay grid function and mmWave path loss model. Relay mechanism works depending on the locations of multicast users to maximize the sum rate.

Extensive simulation results demonstrate that the proposed algorithm improves the average overall throughput compared to the conventional non-relay single-hop directional multicast procedure and delay constraint problem appeared in transmitting videos with high-rate transmission rates in multihop wireless networks.

Ajorloo et al. [32] evaluated the performance of a UWB MAC protocol utilized in WPAN depending on DRP. The authors deduced many formulas to find (1) throughput and size of optimum payload required to obtain the maximum throughput, and (2) starvation and fairness of the protocol.

The experimental results show that the DRP protocol is unfair in some situations. Another enhanced version of the DRP protocol called fair DRP protocol FDRP is proposed. The performance of the modified model is measured in terms of BER, incoming frame rate, payload size, antenna disturbance rate, antenna beam width, network size, network area, and transmission range. Simulations results confirmed the analytical results of the suggested model and showed that FDRP has a little effect on average throughput. On the other hand, it has perfect fairness with approximately zero starvation.

Kim et al. [33] proposed a Multi-Channel Scheduling (MCS) algorithm to solve the conflicts resulting from interfering multiple adjacent Wi-Media logical link protocol Service Sets (WSSs). The proposed MCS algorithm depends on scanning new idle channels and detects its offset time. In addition, the WSS devices transmit new beacon and data frames without conflicts. Channel utilization can be maximized via allowing MCS to utilize all of the dynamically available channels by scanning the channel during the idle 
period. Moreover, the proposed protocol does not require any hardware complexities to avoid interference. Therefore, it can be used in Wi-Media and WUSB standard. The main drawback of the proposed algorithm is causing battery drain when the bridge is a mobile device.

The efficiency of the proposed algorithm was verified using simulation. The simulated results show that MCS algorithm minimizes the number of collisions by efficiently managing the several available channels in a mixed manner utilizing both proactive and reactive methods together.

Kim et al. [34] proposed an adaptive algorithm, called Distributed Satisfaction Ratio of QoS (D_SoQ) to improve WiMedia MAC protocol fairness in a distributed manner. The method is proposed to support high quality of service to each traffic stream in a beacon and to guarantee fair distributed resource allocation. The proposed protocol assumes high rates of traffic and service.

The authors introduced formulas to evaluate the average number of traffic stream and the average delay. The proposed D_SoQ method offers maximum and fair QoS to all traffic streams. Simulation confirmed the numerical results in enhancing traffic stream capacity and achieving a high level of fairness in terms of QoS.

Ayub et al. [35] proposed an algorithm specifically for WSNs called Pilot Assisted Medium Access Control (PAMAC). Authors discussed the reasons which make PA-MAC fails in heavy environments. The proposed algorithm depends on clustering nodes (sensors) in terms of its transmission range from a base station. Cluster membership was determined according to a fixed threshold level. The number of nodes in any cluster never exceeds a predefined limit according to a threshold level.

A cluster called the head cluster was chosen according to a cost function. The cost function was determined according to many parameters such as distance and energy level. Different channels are allocated to different clusters to prohibit intrusion between nodes communicating inside the same clusters (intercluster communication) and those communicating outside the cluster to a base station (intra-cluster communication). The simulation studies show that the efficiency of the PA-MAC in terms delivering of packets, power consumption, and packet drop.

\section{COMPARISONS AND FUTURE WORKS}

This section consists of two parts. In the first part, we compare the different works according to various parameters, while the second part addresses the proposed works to improve UWB applications.

To compare between different previous works, we consider the following parameters:

1) Application: Wireless Body Network (B), Wireless Sensor Network (S), Wireless Personal Area Network (P).
2) Cast: Single Cast (S), Multi-Cast (M), Broadcast (B).

3) Protocol Centralization: Centralized (C), Distributed (D).

4) Number of Hops: Single Hop (S), Multi-Hops (M).

5) Mobility: Fixed (F), Mobile (M).

6) The number of used channels: Single (S), Multiple (M).

7) Model Uniformity: Uniform (U), Non-Uniform (N).

8) Allowing Priority: Yes (Y), No (N).

9) Analytical Approach: Simulation (S), Mathematical (M).

10)Performance Metrics (Commonly Used): Throughput (T), Delay (D), Power Consumption (P), BER (B).

Table I lists the recent works for UWB MAC protocols according to the ten parameters. Based on the data listed in Table I, we introduce some research tracks to be attacked soon in different fields to explore more advances in either analysis or improvements. These suggestions are as follows:

1) Decreasing energy consumption and decreasing throughput are objectives of many research fields on WBANs and WSNs. Therefore, many works plan to evaluate network performance in the case of unsaturated and saturated traffic with low power consumption.

2) Many works did not utilize the characteristics features of UWB signals, e.g. precise position/timing location. Therefore, a considerable effort should be paid to perfectly using these features.

3) An amazing research work can address hot spot traffic where a device or component is targeted by a source more than the others. Also, one or more nodes may be active than the other sources. Introducing such assumptions to WBANs, WSNs or WPANs may reflect more realistic situations. Modeling these cases may be disturbing.

4) Studying different backoff algorithms to both WPANs and WBANs for different operating conditions is a substantial objective for future works. Building participation WPAN antiblocking communication models and redesigning the link scheduling schemes are suggestions of future works. A special interest should be pointed to transmitting videos in multi-hop WPANs.

5) A wonderful and missionary research goal is to study the interference of UWB signals with other signals and how to change the data between UWB WPAN or UWB WBAN and other networks using other signals. For instance, how to change the data between cellular networks and UWB WPANs, UWB WSNs, or UWB WBANs can be transmitted.

6) Important research domains either in UWB networks or other networks are to consider node failure and masked node problems. Therefore, it is a good research goal to handle these problems. Fault tolerance and masked node problem are rich and hopeful research areas. 
TABLE I. SUMmary OF THE ADDRESSED CRITERIA AND PERFormanCE METRICS

\begin{tabular}{|c|c|c|c|c|c|c|c|c|c|c|}
\hline & Application & $\begin{array}{l}\text { Cast } \\
\text { (S-M-B) }\end{array}$ & \begin{tabular}{|l} 
Protocol \\
Centralization
\end{tabular} & $\begin{array}{l}\text { No. of } \\
\text { Hops }\end{array}$ & Mobility & $\begin{array}{l}\text { No. of } \\
\text { Channels }\end{array}$ & Uniformity & Priority & Analysis & $\begin{array}{l}\text { Performance } \\
\text { Metrics }\end{array}$ \\
\hline $\begin{array}{l}{[18]} \\
\end{array}$ & B-S & $M$ & $\mathrm{D}$ & $\mathrm{M}$ & $\mathrm{F}$ & $\mathrm{S}$ & $\mathrm{U}$ & $\mathrm{Y}$ & M-S & T-D-P \\
\hline [27] & $\mathrm{B}$ & $\mathrm{S}$ & $\mathrm{D}$ & $\mathrm{S}$ & $\mathrm{F}$ & $\mathrm{M}$ & $\mathrm{U}$ & $\mathrm{N}$ & M-S & D-P \\
\hline$[28]$ & $\mathrm{B}$ & $\mathrm{S}$ & $\mathrm{C}$ & $\mathrm{S}$ & $\mathrm{M}$ & $\mathrm{M}$ & - & $\mathrm{N}$ & M-S & T-B-P \\
\hline$[8]$ & $\mathrm{B}$ & $\mathrm{S}$ & $\mathrm{D}$ & $\mathrm{S}$ & F-M & $M$ & - & $\mathrm{Y}$ & $\mathrm{S}$ & $\mathrm{P}$ \\
\hline [15] & $\mathrm{B}$ & $\mathrm{S}$ & $\mathrm{D}$ & $\mathrm{M}$ & $\mathrm{M}$ & $\mathrm{S}$ & $\mathrm{U}$ & $\mathrm{N}$ & M-S & - \\
\hline [17] & B-S & - & C-D & S-M & $\mathrm{F}$ & $M$ & - & $\mathrm{N}$ & $\mathrm{S}$ & D-P \\
\hline [16] & B-S & $\mathrm{S}$ & $\begin{array}{l}\mathrm{C} \\
\text { (CSMA/CA) } \\
\text { D( TDM })\end{array}$ & S & $\mathrm{F}$ & M & $\mathrm{U}$ & Y & M-S & T-D-B \\
\hline [29] & B-S & $\mathrm{S}$ & $\mathrm{D}$ & $\mathrm{S}$ & $\mathrm{F}$ & $\mathrm{S}$ & $\mathrm{U}$ & $\mathrm{N}$ & $\mathrm{S}$ & T- D \\
\hline [26] & $\mathrm{P}$ & $\mathrm{S}$ & $\mathrm{D}$ & $\mathrm{S}$ & $\mathrm{F}$ & $\mathrm{M}$ & $\mathrm{N}$ & $\mathrm{N}$ & $\mathrm{S}$ & T-P \\
\hline$[30]$ & $\mathrm{P}$ & $\mathrm{S}$ & $\mathrm{C}$ & $\mathrm{S}$ & $\mathrm{F}$ & $\mathrm{M}$ & $\mathrm{N}$ & $\mathrm{N}$ & M-S & T-P \\
\hline [31] & $\mathrm{P}$ & $\mathrm{M}$ & $\mathrm{C}$ & $\mathrm{M}$ & $\mathrm{F}$ & $\mathrm{S}$ & $\mathrm{N}$ & $\mathrm{N}$ & M-S & T-D \\
\hline [32] & $\mathrm{P}$ & $\mathrm{S}$ & $\mathrm{D}$ & $\mathrm{S}$ & $\mathrm{F}$ & $\mathrm{S}$ & $\mathrm{U}$ & $\mathrm{Y}$ & M-S & $\mathrm{T}$ \\
\hline [33] & $\mathrm{P}$ & M & $\mathrm{D}$ & $\mathrm{M}$ & M & $M$ & $\mathrm{~N}$ & $\mathrm{Y}$ & $\mathrm{S}$ & D-B-P \\
\hline [34] & $\mathrm{P}$ & - & $\mathrm{D}$ & $\mathrm{M}$ & $\mathrm{F}$ & $M$ & $\mathrm{~N}$ & $\mathrm{Y}$ & M-S & $\mathrm{T}$ \\
\hline [35] & $\mathrm{S}$ & $\mathrm{S}$ & $\mathrm{D}$ & $\mathrm{M}$ & $\mathrm{F}$ & $\mathrm{M}$ & $\mathrm{U}$ & $\mathrm{Y}$ & $\mathrm{S}$ & T-P \\
\hline
\end{tabular}

\section{CONCLUSION}

In this article, we introduced the recent advances in UWB applications and performance analysis of MAC protocols for WBANs and WPANs. We addressed the main assumptions and the evaluated performance metrics. Also, we summarized the main contributions of these works and compared between them in terms of ten parameters: application domain, cast type, protocol centralization, number of hops, mobility, number of used channels, uniformity, priority, and analytical approach.

We discovered cases where authors assumed ideal assumptions in contrast to the real situations which are not ideal. Also, many situations were neglected in analyzing the suggested approaches. For example, Fault tolerance and masked node problem were not addressed. According to the interference between different types of communication waves and communicating networks, suitable approaches should be introduced to perfectly utilize the available waves and networks.

Aiming to overcome these situations, we draw a roadmap to a wide area of the research domain. Many research ideas to get more performance in UWB applications or more accuracy in analysis according to different parameters we listed. The listed research ideas may introduce more efficient analysis and modelling techniques or more efficient approaches to the existing applications.

\section{ACKNOWLEDGMENT}

This research was supported by Research Deanship, Hail University, KSA, on grant \#0150431.

\section{REFERENCES}

[1] Y. Mei, W. Aijun, C. Yang and S. Lei, "Photonic Generation of High Order UWB Signals," Optik - International Journal for Light and Electron Optics, Vol. 124, No. 12, Pp. 1079- 1082, 2013.

[2] L. Frank, T. Dimitri and B. Sergio, "Wireless in-Cabin Communication for Aircraft Infrastructure," Telecommunication Systems, Vol. 52, No. 2, Pp. 1211-1232, 2013.
[3] Syed Aftab Naqvi, "Miniaturized Triple-Band and Ultra-Wideband (UWB) Fractal Antennas for UWB Applications," Microwave and Optical Technology Letters, Vol. 59, No. 7, Pp. 1542-1546, 2017.

[4] T. Ming-Chun, X. Shaoqiu, D. Tianwei, W. Duo, G. Jian, W. Bingzhong and G. Guang-Ding, "Compact UWB Antenna with Multiple BandNotches for Wimax and WLAN," IEEE Transactions on Antennas and Propagation, Vol. 59, No. 4, Pp. 1372-1376, 2011.

[5] F. Caglar, E. Serhat and E. C. Mehmet, "Implementation of IEEE 802.15.4a Based UWB Systems for Coexistence with Primary Users," International Journal of Advances in Telecommunications, Electrotechnics, Signals and Systems, Vol. 5, No. 2, Pp. 86-94, 2016.

[6] Z. Jinyun, V. O. Philip, K. Patrick, S. Zafer and F. M. Andreas, "UWB Systems Forwireless Sensor Networks," Proceedings of The IEEE, Vol. 97, No. 2, Pp. 313-331, 2009.

[7] M. Marina, T. Arno, T. Emmeric, V. Luigi, V. Günter, J. Wout, R. Hendrik and M. Luc, "Diversity Performance of Off-Body MB-OFDM UWB-MIMO," IEEE Transactions on Antennas and Propagation, Vol. 63, No. 7, Pp. 3187-3197, 2015.

[8] K. Seungku and K. S. Byung, "A Prioritized Resource Allocation Algorithm for Multiple Wireless Body Area Networks," Wireless Networks, Vol. 23, No. 7, Pp. 727-735, 2017.

[9] S. Alireza, V. Shirmohammadli and M. C. E. Yagoub, "A 3-6 Ghz Current Reused Noise Canceling Low Noise Amplifier for WLAN and WPAN Applications," Wireless Personal Communications, Vol. 86, No. 3, Pp. 1359-1376, 2016.

[10] S. Xiaolin, "A Pulse Design Method for the Compatibility of UWB and IEEE 802.11a WLAN Systems," 2011 International Conference on Network Computing and Information Security, Pp. 130-133, 2011.

[11] R. M. Santosh and S. K. Rakhesh, "Impact of UWB Interference on IEEE 802.11a WLAN System," 2010 National Conference on Communications (NCC), Doi:10.1109/NCC.2010.5430217, IEEE, 2010.

[12] J. Bellorado, S.S. Ghassemzadeh, L. J. Greenstein and V. T. T. Sveinsson, "Coexistence of Ultra-Wideband Systems with IEEE-802.11 a Wireless LANs," Global Telecommunications Conference, Globecom 2003, Vol. 1, Pp. 410-414, 2003.

[13] A. Mason, H. Martin and T. R. Haifa, "Integrating Dual Antennas in to the Wimedia UWB," 9th International Symposium on Communication Systems, Networks \& Digital Sign, Pp. 282-286, 2014.

[14] H. Xiaoan and K. Ryuji, "60-Ghz Ultra-Wideband Radio-Over-Fiber System Employing SCM/WDM," IEEE International Conference on Ultra-Wideband (ICUWB), Pp. 85-90, 2013.

[15] G. Arturo, G. Claire and G. Jean-Marie, "Performance of IR-UWB Cross-Layer Ranging Protocols Under On-Body Channel Models with Body Area Networks," Annals of Telecommunications, Vol. 71, No. 910, Pp. 453-463, 2016. 
[16] K. Vivek and G. Bharat , "Performance Analysis of IEEE 802.15.6 CSMA/CA Protocol for WBAN Medical Scenario Through DTMC Model," Journal of Medical Systems, Vol. 40, No. 12, Pp. 276-291, 2016.

[17] E. A. Maryam, T. Ahmed, L. Rachid and E. M. Nadya, "Impact of Wireless Channel Model on 802.15.6 Standard Performance for Wireless Body Sensor Networks," International Journal of Computer Network and Information Security, Vol. 5, No. 5, Pp. 1-8, 2016.

[18] K. Pervez, U. Niamat, A. Farman, U. Sana, H. Youn-Sik, L. Ki-Young and K. Hoon, "Performance Analysis of Different Backoff Algorithms for WBAN-Based Emerging Sensor Networks," Sensors, Vol. 17, No. 3, 492, 2017.

[19] L. Xuesong and S. Haoshan, "A Location-Aware Ultra-Wideband Based on Dynamic Source Routing Protocol," 2010 International Conference on Computer Application and System Modeling (ICCASM 2010), Vol. 9, DOI: 10.1109/ICCASM.2010.5623147, Pp. 549-553, 2010.

[20] E. Brahim, S. Rachid, E. F. Sanaa, A. Driss and W. Mohamed, "UWB Based Maximizing Network Liftime With Route Selection Strategies for Wireless Sensor Networks," 2011 International Conference on Multimedia Computing and Systems, Pp. 1-7, 2011.

[21] O. Alina, X. Yang and Z. Yan, "Optimization Between AES Security and Performance for IEEE 802.15.3 WPAN," IEEE Transactions on Wireless Communications, Vol. 8, No. 12, Pp. 6030-6037, 2009.

[22] S. Panagiotis, K. Eirini and A. E. Anastasios, "Detecting Sybil Attacks in Wireless Sensor Networks Using UWB Ranging-Based Information," Expert Systems with Applications, Vol. 42, No. 2, Pp. 7560-7572, 2015.

[23] D. Hans, W. Cedric, D. S. Valentijn, D. R. Christophe, M. V. Soheil Radiom, S. Michiel, D. Wim and G. Georges, "A Remotely-Powered, $20 \mathrm{Mb} / \mathrm{S}, 5.35 \mathrm{Pj} / \mathrm{Bit}$ Impulse-UWB WSN Tag for Cm-AccurateLocalization Sensor Networks," Analog Integrated Circuits and Signal Processing, Vol. 80, No. 3, Pp. 531-540, 2014.

[24] C. Abdellah, F. Paul and M. T. Pierre, "UWB-Based Sensor Networks for Localization in Mining Environments," Ad Hoc Networks, Vol. 7, No. 5, Pp. 987-1000, 2009.

[25] T. Mostafa and A. M. Seyed, "Energy-Efficient Cooperative Localization in Mobile WSN," IEEE Transactions on Electrical and
Electronic Engineering, Vol. 12, No. 1, Pp. 71-79, 2017.

[26] J. Yang-Ick and H. Kyeong, "Cooperative Distributed Mac Design for Cross-Layer Link Adaptation of UWB WPAN Devices," Wireless Personal Communications, Vol. 71, No. 1, Pp. 137-150, 2013.

[27] W. Zhongcheng, S. Yongmei and J. Yuefeng, "A Study on Coexistence Capability Evaluations of the Enhanced Channel Hopping Mechanism in WBANS," Sensors, Vol. 17, No. 1, Pp. 151-186, 2017.

[28] S. Miftadi, T. Le Chung, S. Farzad, W. Tadeusz and L. P. Son, "HighSpeed Adaptive Wireless Body Area Networks," Eurasip Journal on Wireless Communications and Networking, Vol. 2016, No. 1, 296, 2016.

[29] D. Jin , Z. Li, M. Sirisha and M. S. Krishna, "Mac Protocols for UltraWide-Band (UWB) Wireless Networks: Impact of Channel Acquisition Time," Proc. Spie 4869, Emerging Technologies for Future Generation Wireless Communications, Doi: 10.1117/12.455471, 2002.

[30] S. Wei, W. Jingjing, Z. Hao, L. Yun, N. Qiuna and W. Chunlei, "A New Link Scheduling Algorithm for 60 Ghz-WPAN Communication System," International Journal of Distributed Sensor Networks, Vol. 2016, Id:6395385, 2016.

[31] P. Hyunhee, P. Seunghyun, S. Taeshik and K. Eui-Jik, "Multi-HopBased Opportunistic Concurrent Directional Transmission in $60 \mathrm{Ghz}$ WPANs," Multimedia Tools and Applications, Vol. 74, No. 5, Pp. 1627-1644, 2015.

[32] A. Hossein and M.T. Manzuri-Shalmani, "Throughput Modeling of Distributed Reservation Protocol," IEEE Transactions on Mobile Computing, Vol. 15, No. 2, Pp. 503-515, 2016.

[33] K. Jin-Woo, H. Kyeong and L. Seong-Ro, "A Multi-Channel Scheduling Scheme for Collision-Free High-Rate WPANs," Wireless Personal Communications, Vol. 78, No. 1, Pp. 429-448, 2014.

[34] K. Seokhwan, H. Kyeong, P. Jongsun, E. Doo-Seop and H. Kwang-Il, "A Fair Distributed Resource Allocation Method in UWB Wireless PANs with Wimedia Mac," Journal of Communications and Networks, Vol. 11, No. 4, Pp. 375-383, 2009.

[35] A. Kamran and Z. Valerijs, "Adoption Features and Approach for UWB Wireless Sensor Network Based on Pilot Signal Assisted MAC," International Journal of Communication Networks and Information Security (IJCNIS), Vol. 8, No. 1, Pp. 40-46, 2016. 